Article

\title{
Suppression of Frequency Modulation to Amplitude Modulation Conversion with Modified Group Velocity Dispersion Compensation Device in the Front End of High-Power Lasers
}

\author{
Xinlei Qian ${ }^{1,2}$, Xiaochao Wang ${ }^{1, *}$, Xinghua Lu ${ }^{1}$, Tianyu Zhang ${ }^{1,2}$ and Wei Fan ${ }^{1,2, *}$ \\ 1 National Laboratory on High Power Laser and Physics, Shanghai Institute of Optics and Fine Mechanics, \\ Chinese Academy of Sciences, Shanghai 201800, China; xlqian@siom.ac.cn (X.Q.); luxingh@siom.ac.cn (X.L.); \\ zhangtianyu@siom.ac.cn (T.Z.) \\ 2 Center of Materials Science and Optoelectronics Engineering, University of the Chinese Academy of Sciences, \\ Beijing 100049, China \\ * Correspondence: smilexc@siom.ac.cn (X.W.); fanweil@siom.ac.cn (W.F.)
}

check for

updates

Citation: Qian, X.; Wang, X.; Lu, X.;

Zhang, T.; Fan, W. Suppression of

Frequency Modulation to Amplitude

Modulation Conversion with

Modified Group Velocity Dispersion

Compensation Device in the Front

End of High-Power Lasers. Appl. Sci. 2022, 12, 884. https://doi.org/

10.3390/app12020884

Academic Editor: Katarzyna Antosz

Received: 17 November 2021

Accepted: 14 January 2022

Published: 16 January 2022

Publisher's Note: MDPI stays neutral with regard to jurisdictional claims in published maps and institutional affiliations.

Copyright: (c) 2022 by the authors. Licensee MDPI, Basel, Switzerland. This article is an open access article distributed under the terms and conditions of the Creative Commons Attribution (CC BY) license (https:// creativecommons.org/licenses/by/ $4.0 /)$.

\begin{abstract}
The group velocity dispersion (GVD) occurring in the front end of high-power lasers is one of the primary factors leading to the conversion of frequency modulation (FM) to amplitude modulation (AM). In this paper, we propose a modified, active, closed-loop feedback compensation device for GVD-induced FM-AM conversion, using a two-dimensional, electric, adjustable mirror mount and parallel grating pair to improve the long-term stability, efficiency of adjustment, and accuracy of compensation. Experimental results of a $12 \mathrm{~h} \mathrm{FM-AM}$ depth test revealed that the depth varied between $2.28 \%$ and $5.22 \%$. Moreover, we formulated a mathematical relationship between the dispersion parameters and temperature in optical fibers to analyze the intrinsic effect of temperature on FM-AM. The related simulation and experimental results consistently validated the quantitative relationship between the temperature and FM-AM depth.
\end{abstract}

Keywords: dispersion compensation devices; lasers; frequency modulation; amplitude modulation

\section{Introduction}

A high-power laser driver is an important component of an inertial confinement fusion (ICF) laser system, composed of a front end, preamplifier, main amplifier, target, and other control and diagnostic systems [1,2]. For high-power laser pulses in the nanosecond regime, spectral broadening in large-scale laser facilities is an essential method to suppress transverse stimulated Brillouin scattering (TSBS) [3]. TSBS is a three-wave coupled nonlinear process, where the incident light energy is converted into transverse scattered light energy, resulting in energy loss; simultaneously, a strong elastic acoustic field is generated in the medium, causing mechanical damage to the optical components. However, increasing the spectral bandwidth can help reduce the threshold of TSBS [4]. In addition, smoothing via spectral dispersion (SSD) is used to smooth the speckle pattern illuminating the target; this also requires the high-energy laser to be spectrally broadened in the time domain [5]. Typically, a low-frequency phase modulation is used to broaden the spectrum of the seedsource laser to reduce the power spectral density, and a high-frequency phase modulator is used to achieve the optical smoothing of the focal spot to meet the physical shooting requirements [6,7]. Under ideal conditions, when transmitting after phase modulation, the pulse retains its original time-domain characteristics and the spectrum is uniform [8]. Actually, the spectral transfer functions of all the optical components are nonuniform, which is caused by the group velocity dispersion (GVD), polarization dispersion, change in the index of refraction, and nonuniform spectral transmission from clipping by the etalon effects [9]. The spectrum of the phase-modulated broadband light is affected, leading to 
the generation of FM-AM, when passing through these optical elements with nonuniform spectral transmittance [10]. If no measures are adopted to suppress or compensate for this, FM-AM will influence the power balance of the laser drive and damage the optical components during transmission [11,12].

In the front end of high-power lasers, the main factors influencing the generation of FM-AM are the polarization mode dispersion (PMD), GVD, and gain-narrowing effect of the regenerative preamplifier [13,14]. Many researchers have proposed related technical schemes. To eliminate the effect of PMD, Z. Qiao proposed using a high-extinction-ratio single polarization [15]. J.T. Guo demonstrated a broadband Nd:glass regenerative laser amplifier system with an intracavity birefringent filter to suppress FM-to-AM conversion [16]. M.Q. Fan used a two-dimensional tunable and temperature-insensitive Lyot filter for compensation [17]. The transmission peak shift was less than that under a normal polarization maintaining fiber (PMF) configuration. Therefore, the GVD is the most important factor influencing the FM-AM conversion for the front end [18]. As we all know, the effect of GVD can be negligible over short fiber distances; however, long fiber distances of several hundred meters are required for transmission in front-end systems, which can produce severe distortions [19]. In addition, J. Kim proposed that chirped fiber Bragg gratings (CFBGs) can achieve a better GVD compensation effect, owing to their flexible implementation, low loss, and weak nonlinearity [20]. However, the performance of CFBGs is easily affected by environmental factors. Furthermore, CFBGs introduce a large amount of dispersion and are often used to compensate for long transmission fibers (several kilometers) in communication systems. The high-energy laser facilities, such as the National Ignition Facility (NIF) and Laser Mégajoule Facility (LMJ), preferentially use bulk gratings to compensate for GVD [21,22]. Depending on the synchronization requirements, the injected optical parameters may vary for different fiber lengths; thus, the compensation process needs to be reconstructed, which makes the process time inefficient and time-consuming. Therefore, stable, tunable, and efficient GVD compensation is necessary for high-power laser facilities.

In this paper, we propose a modified, active, closed-loop feedback compensation device for GVD-induced FM-AM conversion using a two-dimensional, electric, adjustable mirror mount and parallel grating pair to improve the long-term stability and efficiency. The experimental results showed that the influence of FM-AM is notably suppressed in long-term testing; the depth ranged from approximately $2.28 \%$ to $5.22 \%$. Compared with manual regulation, the motor closed-loop feedback control affords higher accuracy, stability, and efficiency of adjustment. Moreover, the depth drifts slightly at the laboratory temperature, which cannot be fully compensated. The relationship between the depth and temperature was quantified via simulation calculations and was in agreement with the drift observed in the experiment.

\section{Principle and System Structure}

\subsection{Principle of GVD Compensation}

GVD compensation technology is very mature; the commonly used schemes are mainly divided into two types, including chirped fiber grating compensation and solid grating-pair compensation techniques. Among the two compensation schemes, chirped fiber grating introduces a larger dispersion, and is usually used to compensate for the long transmission fibers (several kilometers) in communication systems. By contrast, parallel grating pairs introduce a smaller dispersion and are more suitable for the GVD compensation of short transmission fibers. The length of the transmission optical fiber used in the system is $50 \sim 200 \mathrm{~m}$, the dispersion coefficient is $18.7 \mathrm{ps}^{2} / \mathrm{km}$, and the introduced dispersion is approximately $0.94 \sim 3.74 \mathrm{ps}^{2}$. Solid gratings are more stable and less susceptible to environmental influences than fiber gratings; therefore, the solid grating was chosen for the GVD compensation scheme.

The principle of parallel grating-pair compensation is illustrated in Figure 1. The incident light was dispersed by the grating. By selecting an appropriate incident angle and grating parameters, the long-wavelength component takes longer to pass through the 
grating pair than the short-wavelength component. Thus, the long-wavelength component is output after the short-wavelength component on passing through the grating pair; in other words, a negative GVD is introduced to offset the positive GVD introduced by the transmission fiber to realize compensation. As shown in Figure $1, \gamma$ is the angle of incidence, $\alpha$ is the angle of diffraction, and $G$ is the vertical spacing of the grating pair. The path of a monochromatic light with a frequency component $\omega$ in the grating pair is [23]

$$
x(\omega)=\frac{G\{1+\cos [\gamma-\alpha(\omega)]\}}{\cos [\alpha(\omega)]}
$$

where $\alpha(\omega)$ denotes the frequency functions satisfying the grating equation

$$
\sin \gamma+\sin [\alpha(\omega)]=\frac{2 \pi c}{w d}
$$

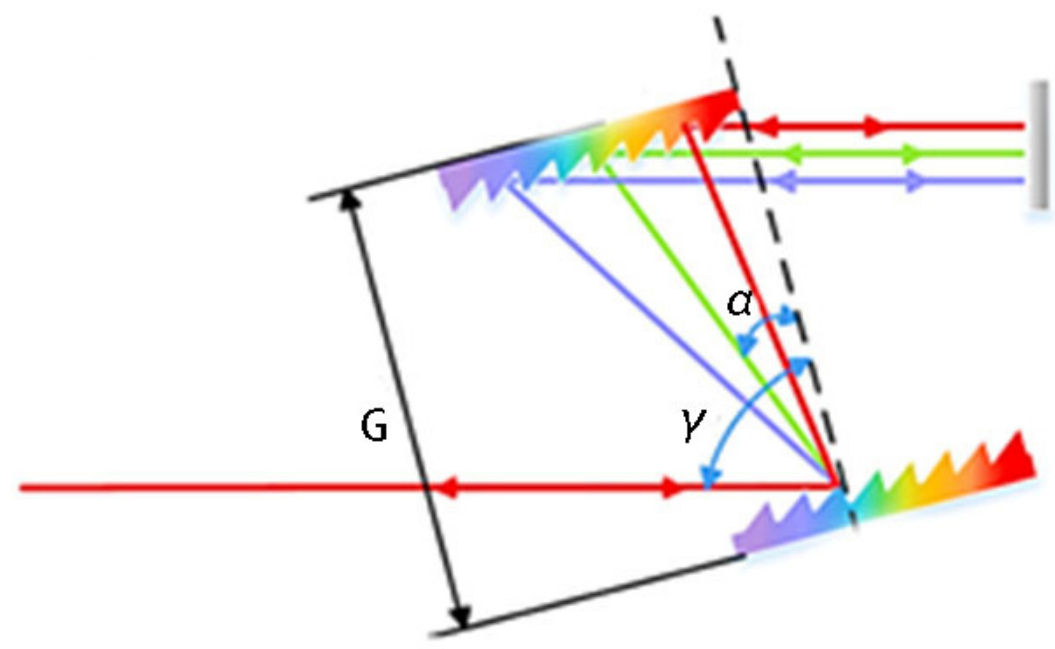

Figure 1. Principle diagram of dispersion induced by parallel grating pairs.

When the signal light is diffracted at different positions of the grating, the phase delay satisfies:

$$
\varnothing(\omega)=\left(\frac{\omega}{c}\right) x(\omega)-\frac{2 \pi G}{d} \tan [\alpha(\omega)]
$$

where $c$ is the speed of light and $d$ is the grating constant. For nanosecond pulses, the effect of higher-order dispersion can usually be neglected and only the second-order dispersion needs to be considered. By substituting Equation (1) into Equation (3) and performing derivation, the second-order dispersion $D$ introduced by the parallel grating pairs can be obtained

$$
D=\frac{G \lambda}{c \cos [\alpha(\lambda)]^{3} d^{2}}
$$

where $\lambda$ is the wavelength. The amount of dispersion can be tuned by varying the grating constant $d$, grating-pair spacing $G$, and angle of incidence $\gamma$ for parallel grating pairs.

For nanosecond pulses, although GVD does not cause significant pulse spreading, the strong GVD induced by long transmission fibers in a system can lead to severe amplitude and frequency modulation, resulting in waveform distortion.

After long fiber transmission, the output optical power $P_{2}$ can be expressed as:

$$
P_{2}=P_{1}\left\{1-\beta_{2} L\left[m_{1} \omega_{1}^{2} \sin \left(\omega_{1} T\right)+m_{2} \omega_{2}^{2} \sin \left(\omega_{2} T\right)\right]\right\}
$$

where $P_{1}$ is the input optical power, $L$ is the fiber length, $m_{1}$ and $m_{2}$ are the depths of the two levels of phase modulation; $\omega_{1}$ and $\omega_{2}$ are the modulation frequency of the two levels of phase modulation; $\beta_{2}$ is the GVD; and T is the relative time of the pulse envelope 
of the GVD. From Equation (5), the frequency modulation is partially converted into the amplitude modulation of the power. The amplitude modulation takes the same functional form as the frequency modulation, and the modulation depth increases with $\beta_{2} L$.

To determine the numerical magnitude of FM-AM, the depth can be expressed as:

$$
\alpha=\frac{I_{\max }-I_{\min }}{\left(I_{\max }+I_{\min ) / 2}\right.}
$$

where $\alpha$ is the FM-AM depth that ranges from $0 \%$ to $200 \%$. The terms $I_{\max }$ and $I_{\min }$ are the maximal and minimal intensities of the time waveform, respectively [24].

\subsection{Structure of GVD Compensation}

Typically, $d$ is constant and $G$ is an inconvenience adjustment. The slight non-parallelism introduces a spatial chirp, which is detrimental to subsequent coupling. The most appropriate method to regulate compensation dispersion is to adjust the two-dimensional orientation of the plane mirror to alter the diffraction angle. However, manual adjustment is not sufficiently accurate nor efficient, and cannot be applied using adaptive feedback. Hence, an electric adjustment is required for precise tuning and active closed-loop feedback compensation. A schematic of the active closed-loop feedback compensation scheme is presented in Figure 2. The pulse with phase modulation attains FM-AM after transmission, which can be reduced after compensation. The oscilloscope then collects the time-domain signal and transmits it to the computer to calculate the corresponding FM-AM depth. If the value of the depth increases owing to external interference or changes in the input light parameters, according to the recorded data, a control signal is generated by a computer program and fed back to the mirror motor control, and then automatically fine-tuned to improve the compensation results.

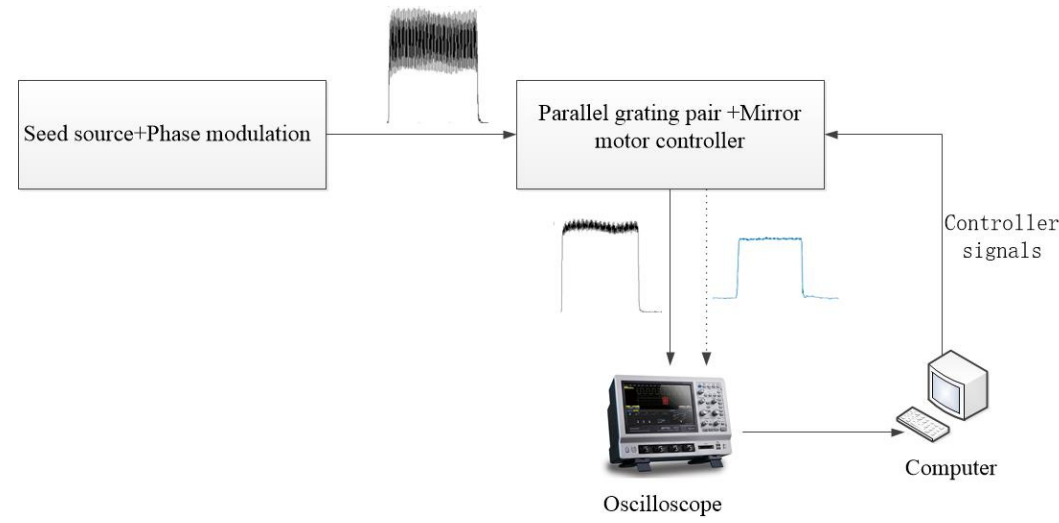

Figure 2. Schematic of compensation device.

\section{Experimental Steps and Results}

A schematic and a photograph of the tunable dispersion compensator are shown in Figure 3. This device includes a fiber circulator, a fiber collimator, a half-wave plate, two blazed gratings with identical parameters $(40 \mathrm{~mm} \times 40 \mathrm{~mm}, 1740 \mathrm{gr} / \mathrm{mm}$, diffraction wavelength of $1060 \mathrm{~nm}$, diffraction efficiency of $86 \%$ at $1053 \mathrm{~nm}$, a Littrow angle of $67.2^{\circ}$, and $\mathrm{Au}$ coating), and a two-dimensional electric adjusting mirror mount. The relevant parameters were estimated based on the grating parameters used in the experiment, including size, diffraction efficiency, wavelength, linear density, and spot size of incident light. We think these parameters have good reference value. Thus, in the tuning process, more accurate small-scale adjustment was required. The electric adjusting mirror mount was produced by New Focus. The Type 8742 is a four-axis open-loop intelligent motion controller/driver that serves as a compact and cost-effective solution for driving New Focus open-loop picomotor products. It is a single-box solution that can be computer-controlled using the Newport Motion Control Language (NMCL) command set via USB and Ethernet com- 
munication interfaces. An oscilloscope with a bandwidth of $30 \mathrm{GHz}$ (Agilent, DSO93004 L) and a high-speed photodetector with a bandwidth of $45 \mathrm{GHz}$ (Newport, 1014) were used to collect the nanosecond pulses in the time domain. The injecting spectrum was applied with a two-stage phase modulation $(3 \mathrm{GHz}$ and $20 \mathrm{GHz}$ ) and a $3 \mathrm{~dB}$ bandwidth of $0.3 \mathrm{~nm}$. After the input pulse light was transmitted through the optical fiber, it entered the circulator 1 port and was output from the circulator 2 port into the optical fiber collimator. Subsequently, after collimation and expansion, the beam passed through a half-wave plate and was incident on grating 1 . The diffraction efficiency of the grating is closely related to the polarization state of the incident light. Therefore, it was necessary to rotate the wave plate and adjust the polarization state of the incident light to achieve the highest diffraction efficiency of the grating and reduce the system loss. Next, the light beam was diffracted by grating 1 and transmitted over a certain distance in space to grating 2 . Thereafter, it was diffracted by grating 2 for a second time, became a parallel light beam, reached the plane mirror, was reflected by it, returned to the collimator, was coupled into the optical fiber by the collimator, and re-entered port 2 of the circulator. Finally, the output from port 3 of the circulator entered the next section of the fiber for further transmission. The angle of incidence can be altered by adjusting the two-dimensional direction of the plane mirror to tune the grating's dispersion. The overall insertion loss of the system was approximately $5 \mathrm{~dB}$, mainly originating from the annular insertion loss and coupling. If a low insertion loss circulator is used and the coupling is finely tuned, the system insertion loss can be further reduced. In the experiment, in order to avoid the introduction of additional FM-AM by PMD, a single polarization fiber was used as the transmission fiber. In the ICF driver, for accurate synchronization, the length of the transmission optical fiber used for each channel is usually different, ranging from 50 to $200 \mathrm{~m}$. In addition, the seed spectral width of the front-end system should be adjustable within $0.1 \sim 0.3 \mathrm{~nm}$ and the central wavelength is required to be adjustable within $1053.0 \pm 0.5 \mathrm{~nm}$; this necessitates that the compensation system should be tunable and also possess a certain tolerance for the tuning of the signal spectral width and central wavelength.

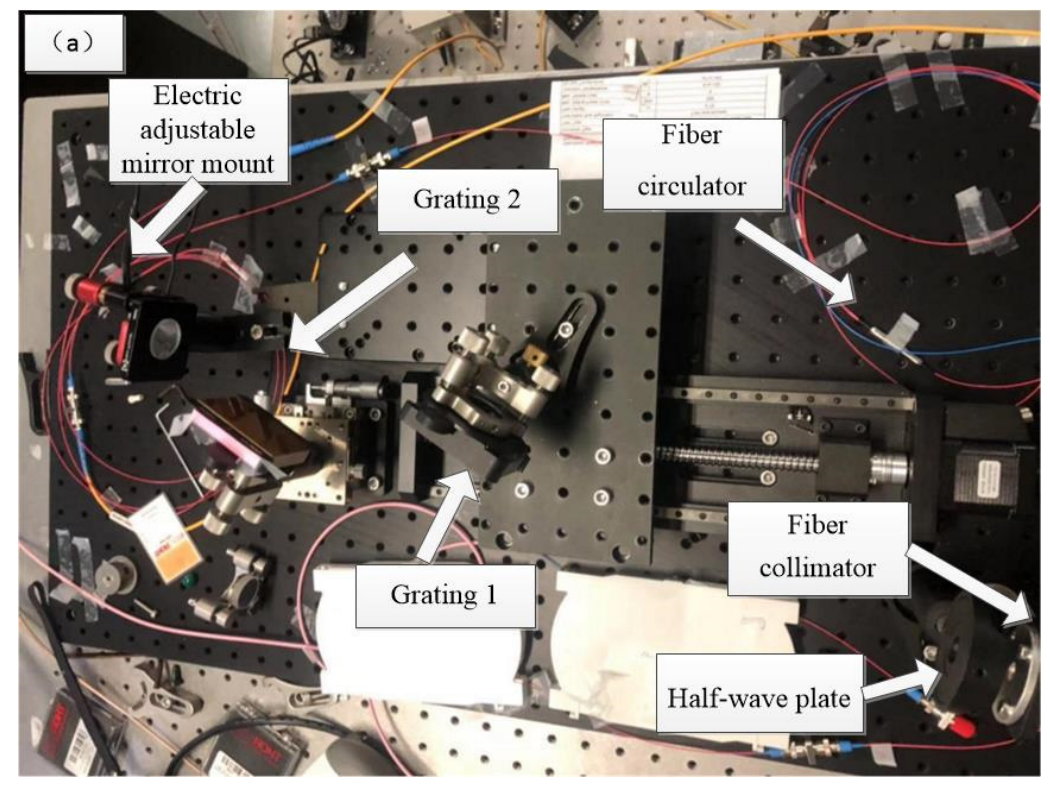

Figure 3. Cont. 


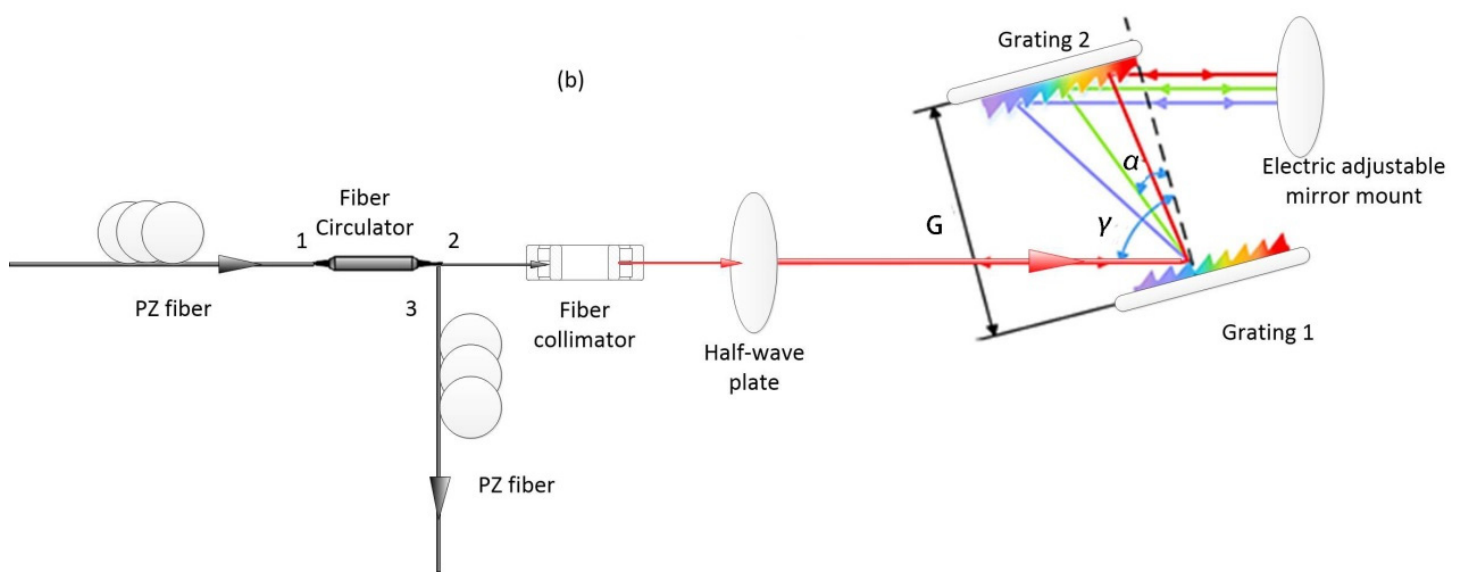

Figure 3. Diagram of compensation device (a) photograph; (b) schematic.

First, we roughly scanned the relationship between the FM-AM depth and the motor operating state, including the direction and steps of the motor. The diagram of the motor scan is shown in Figure 4, where step 1 and step 2 represent the two dimensions of the motor, respectively. The positive and negative values represent the direction and the value represents the steps of the motor adjustment. The ordinate represents the depth of a certain motor state. Scanning was conducted to identify the area with the minimum FM-AM depth quickly and to improve feedback efficiency. The figure is concave toward the center along the circumference; this concave point indicates the minimum FM-AM depth.

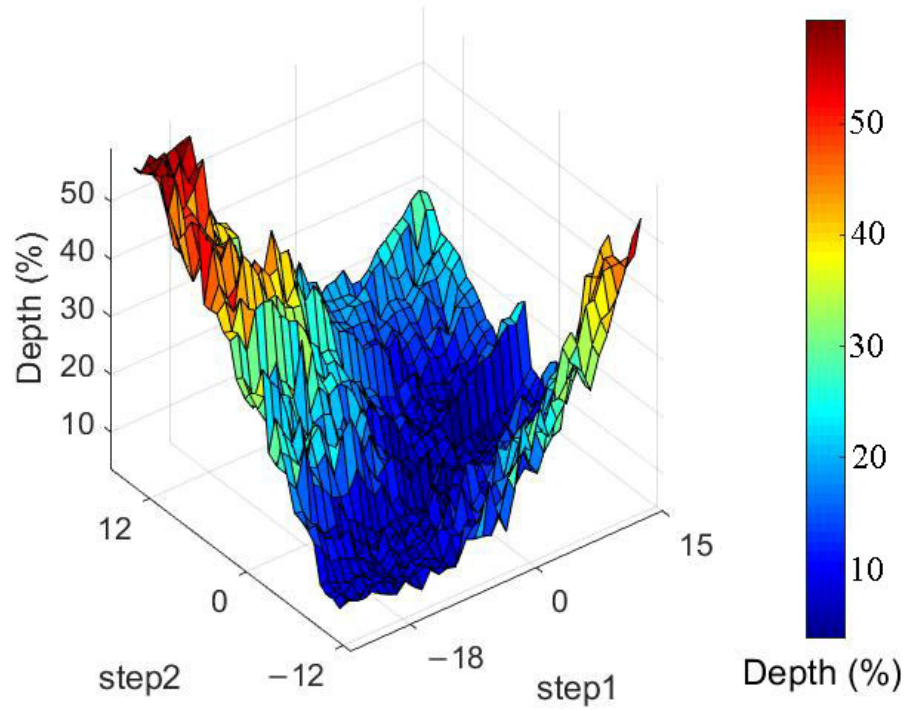

Figure 4. Diagram of motor scan.

The time-domain pulse diagram obtained before and after compensation is shown in Figure 5. The relationship between intensity and depth can be expressed as Equation (6). The pulse featured a significantly large depth before the compensation for dispersion, reaching $96 \%$. By contrast, the depth was reduced to $3.8 \%$ when employing the active closed-loop feedback GVD compensation device. We think that a depth of less than $5 \%$ is a relatively good state, which is also the standard and aim of our experiment and engineering. We can adjust other parameters to further reduce the depth, such as adjusting the grating spacing, but it is difficult to control it manually to meet the adjustment accuracy. Thus, FM-AM can be well suppressed because the compensation system compensates for the GVD of the long transmission optical fiber, and also because of the GVD introduced by the optical fiber in the seed source. 


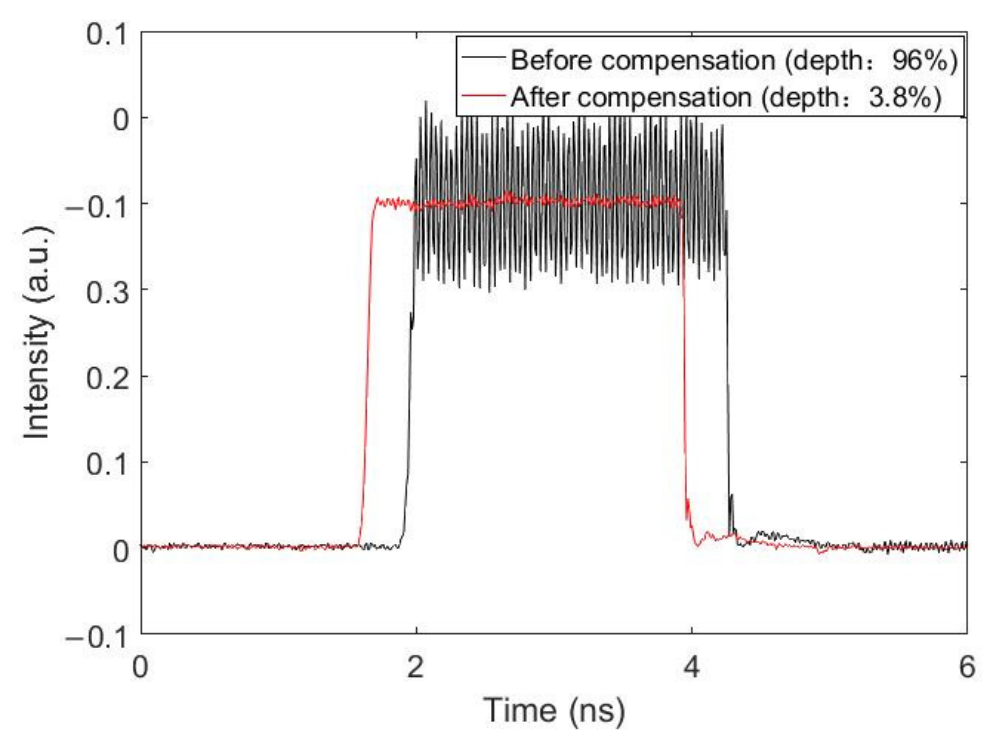

Figure 5. Time-domain pulse diagram before and after compensation.

Figure 6 shows the results of the depth test over $12 \mathrm{~h}$. As indicated by the graph, the depth varied between $2.28 \%$ and $5.22 \%$. This also demonstrates the good long-term reliability of the feedback device. However, certain slight drifts of approximately $0.1 \sim 0.3 \%$ can also be detected in the graph; these can possibly be attributed to the influence of the variations in the laboratory temperature, because temperature is closely related to the dispersion of the fiber.

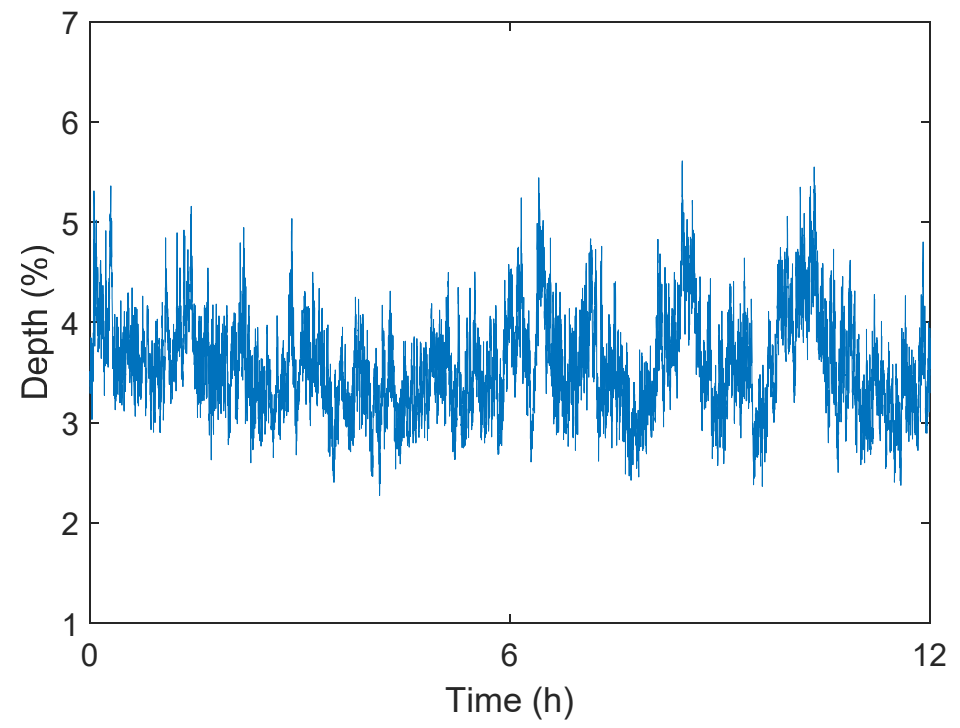

Figure 6. Depth test over $12 \mathrm{~h}$.

Therefore, we recorded the laboratory temperature under the same environmental and time conditions for verification. As shown in Figure 7, the first $6 \mathrm{~h}$ are the night when researchers work in the laboratory. The temperature of the equipment and of the people will cause temperature changes. The next six hours are the night break. The result of the test is unstable, with fluctuations of approximately $0.5 \sim 1.5^{\circ} \mathrm{C}$; this rightfully has a direct impact on the dispersion compensation. The corresponding depth change was approximately $0.3 \%$. Clearly, the change in temperature is a long-term, slow drift, and we think the effect of temperature on the depth is a slow cumulative process, rather than a violent sudden change; additional means are required to overcome this. This will be a focus of our future research. 


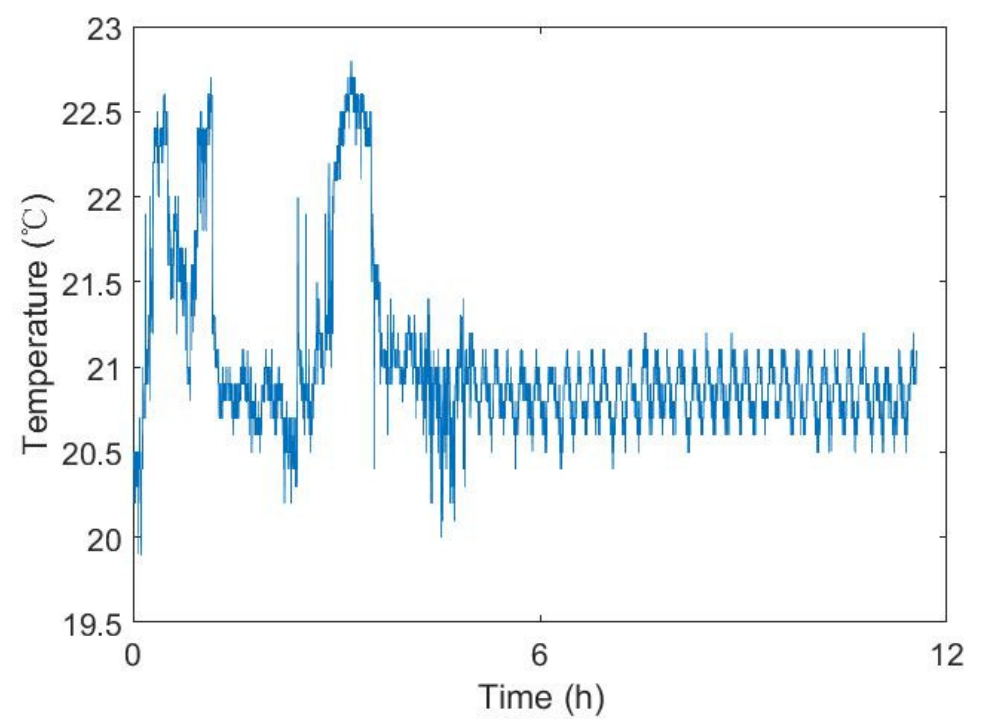

Figure 7. Results of laboratory temperature test over $12 \mathrm{~h}$.

\section{Discussion}

As shown in Figures 6 and 7, the temperature variation is one of the factors influencing FM-AM. Environmental temperature variation can alter the refractive index of a fiber slightly, which, in turn, results in a drift in the dispersion, especially in long transmission fibers. Thus, the values of the system's FM-AM depth vary depending on temperature. For a section of the fiber optic link, the relationship between the dispersion parameter and temperature can be expressed as follows:

$$
\begin{aligned}
D_{T}=\frac{n L a b}{C \lambda} T^{2} & +\frac{n L(a+b)}{C \lambda} T+\frac{n L}{C \lambda} \\
a & =\frac{1}{n} \cdot \frac{\partial n}{\partial T} \\
b & =\frac{1}{L} \cdot \frac{\partial L}{\partial T}
\end{aligned}
$$

where $D_{T}$ is the dispersion parameter, $n$ is the refractive index, $a$ is the thermal light factor, $b$ is the coefficient of thermal expansion, $L$ is the fiber length, and $\lambda$ is the wavelength. We can adopt the following parameters to obtain the $D_{T}$ curve: $L=100 \mathrm{~m}, a=10^{-5} /{ }^{\circ} \mathrm{C}$, and $b=5.5 \times 10^{-7} /{ }^{\circ} \mathrm{C}$. These curves are depicted in Figure 8. Based on the Equation (7), $D_{T}$ varies along a quadratic curve. However, because the thermal expansion coefficient and the thermo-optical coefficient are both considerably small, the product of the two values will be smaller, which can be regarded as a linear function, and the values of dispersion increase almost linearly with the temperature. Because of this linear variation, proportionality is used to equivalently convert the GVD and compare the variation in the FM-AM depth.

As shown in Figure 8, when the temperature was increased from $20{ }^{\circ} \mathrm{C}$ with steps of 1 and $3{ }^{\circ} \mathrm{C}$, the dispersion value increased by $5 \%$ and $15 \%$, respectively. By combining the quantized relationships with the FM-AM simulation process, the depth increases with the temperature rises owing to the variations in the GVD, which are $4.25 \%, 4.46 \%$, and $4.88 \%$. The variation in depth is approximately $0.25 \%$, corresponding to a drift of $1{ }^{\circ} \mathrm{C}$. The results obtained from the simulation were almost identical to the drift noted in the experiment $(0.1 \sim 0.3 \%)$. Therefore, temperature drift is not conducive to compensating dispersion. 


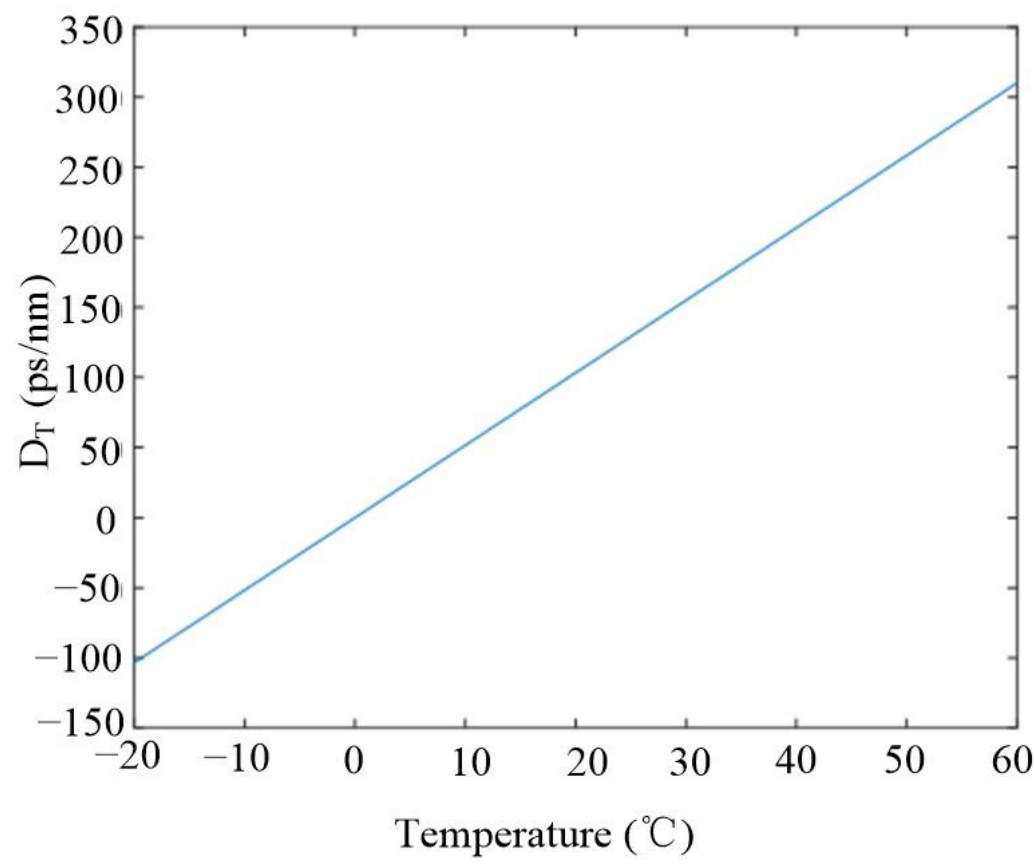

Figure 8. Dispersion parameters at different temperatures.

When adjusting the grating spacing, the theoretical calculation can initially only provide a rough estimate of the grating pair position. Thus, it is necessary to fine-tune the spacing in the experiment via manual adjustments to accurately compensate for the second-order dispersion of the system. If only the distance between the grating pairs is adjusted manually, it is difficult to control the adjustment accuracy; it also difficult to ensure that the angle of the grating is appropriate, which makes it difficult to adjust the optical path. Notably, the scheme of controlling the grating-pair spacing by using an electric guide rail suffers from certain limitations, such as poor robustness, difficultly in adjustments, and high economic costs. Hence, using an electric, adjustable mirror mount and a fixed parallel grating pair is still the better choice for realizing small amounts of automatic dispersion compensation.

\section{Conclusions}

To summarize, in this work, FM-AM modulation was suppressed using a modified, active, closed-loop feedback compensation device with an electric, adjustable mirror mount and a fixed parallel grating pair. The experimental results of a $12 \mathrm{~h}$ FM-AM depth test showed that the depth varied from $2.28 \%$ to $5.22 \%$. We also quantified the relationships between the FM-AM depth and temperature using an FM-AM simulation program and theoretical analyses; a depth change of $0.25 \%$ was found to correspond to a drift of $1{ }^{\circ} \mathrm{C}$. This simulation result is almost consistent with the drift observed in the experiment. We believe that the proposed scheme and relevant theoretical simulation can improve the suppression ability of FM-AM and lead to a more precise time-frequency control capability in the front-end system of high-power laser devices.

Author Contributions: Conceptualization, X.Q. and X.W.; methodology, X.Q.; software, X.L.; validation, X.Q. and X.W.; formal analysis, X.Q. and T.Z.; investigation, T.Z.; resources, W.F.; data curation, W.F.; writing — original draft preparation, X.Q.; writing-review and editing, X.Q.; visualization, W.F.; supervision, W.F.; project administration, W.F. and X.W.; funding acquisition, W.F. and X.W. All authors have read and agreed to the published version of the manuscript.

Funding: This work was supported by the Strategic Priority Research Program of the Chinese Academy of Sciences (No. XDA25020303).

Institutional Review Board Statement: Not applicable. 
Informed Consent Statement: Not applicable.

Data Availability Statement: The data included in this study are all owned by the research group and will not be transmitted.

Acknowledgments: The authors thank the editor and anonymous reviewers for their valuable comments.

Conflicts of Interest: The authors declare no conflict of interest.

\section{References}

1. Dorrer, C.; Roides, R.; Cuffney, R.; Okishev, A.V.; Bittle, W.A.; Balonek, G.; Consentino, A.; Hill, E.; Zuegel, J. Fiber front end with multiple phase modulations and high-bandwidth pulse shaping for high-energy laser-beam smoothing. IEEE J. Sel. Top. Quantum Electron. 2013, 19, 219-230. [CrossRef]

2. Hocquet, S.; Penninckx, D.; Bordenave, E.; Gouédard, C.; Jaouën, Y. FM-to-AM conversion in high-power lasers. Appl. Opt. 2008, 47, 3338-3349. [CrossRef] [PubMed]

3. Kaler, R.; Kama, T.; Sharma, A.; Arya, S.; Agarwala, R. Large signal analysis of FM-AM conversion in dispersive optical fibers for PCM systems including second order dispersion. Fiber Integr. Opt. 2002, 21, 193-203. [CrossRef]

4. Xu, D.; Tian, X.; Zhou, D.; Zong, Z.; Fan, M.; Zhang, R.; Zhu, N.; Xie, L.; Li, H.; Wang, J.; et al. Temporal pulse precisely sculpted millijoule-level fiber laser injection system for high-power laser driver. Appl. Opt. 2017, 56, 2661-2666. [CrossRef] [PubMed]

5. Lindl, J.; Amendt, P.; Berger, R.L. The physics basis for ignition using indirect-drive targets on the national ignition facility. Phys. Plasmas 2004, 11, 339-491. [CrossRef]

6. Penninckx, D.; Beck, N.; Gleyze, J.F.; Videau, L. Signal propagation over polarization-maintaining fibers: Problem and solutions J. Lightwave Technol. 2006, 24, 4197-4207. [CrossRef]

7. Dorrer, C. Spectral and temporal properties of optical signals with multiple sinusoidal phase modulations. Appl. Opt. 2014, 53, 1007-1019. [CrossRef] [PubMed]

8. $\quad$ Bian, Y.; Jiao, K.; Wu, X.; Yang, H.; Zhu, R. Utilizing phase-shifted long-period fiber grating to suppress spectral broadening of a high-power fiber MOPA laser system. High Power Laser Sci. Eng. 2021, 9, e39. [CrossRef]

9. Takabe, H.; Kuramitsu, Y. Recent progress of laboratory astrophysics with intense lasers. High Power Laser Sci. Eng. 2021, 9, e49. [CrossRef]

10. Vidal, S.; Luce, J.; Penninckx, D. Experimental demonstration of linear precompensation of a nonlinear transfer function due to second-harmonic generation. Opt. Lett. 2011, 36, 88-90. [CrossRef]

11. Bowers, M.; Cohen, S.; Erbert, G.; Heebner, J.; Hermann, M.; Jedlovec, D. The injection laser system on the National Ignition Facility. Proc. SPIE 2007, 6451, 64511M.

12. Waxer, L.J.; Kelly, J.H.; Rothenberg, J.; Babushkin, A.; Bibeau, C.; Bayramian, A.; Payne, S. Precision spectral sculpting for narrowband amplification of broadband frequency-modulated pulses. Opt. Lett. 2002, 27, 1427-1429. [CrossRef] [PubMed]

13. Rothenberg, J.E.; Browning, D.F.; Wilcox, R.B. Issue of FM to AM conversion on the National Ignition Facility. Proc. SPIE 1999, 3492, 51-62.

14. Li, R.; Fan, W.; Jiang, Y.; Qiao, Z.; Zhang, P.; Lin, Z. Tunable compensation of GVD-induced FM-AM conversion in the front end of high-power lasers. Appl. Opt. 2017, 56, 993-998. [CrossRef] [PubMed]

15. Qiao, Z.; Wang, X.; Fan, W.; Lin, Z. Demonstration of a high-energy, narrow-bandwidth, and temporally shaped fiber regenerative amplifier. Opt. Lett. 2015, 40, 4214-4217. [CrossRef]

16. Guo, J.; Wang, J.; Pan, X.; Lu, X.; Xia, G.; Wang, X.; Zhang, S.; Fan, W.; Li, X. Suppression of FM-to-AM conversion in abroadband Nd:glass regenerative amplifierwith an intracavity birefringent filter. Appl. Opt. 2019, 58, 1261-1270. [CrossRef]

17. Fan, M.; Tian, X.; Zhou, D.; Wei, J.; Xia, H.; Lv, H.; Zhao, H.; Xu, D.; Zheng, W. Two-Dimensional Tunable and TemperatureInsensitive Lyot Filter for FM-to-AM Compensation. Photonic Sens. 2021, 11, 325-333. [CrossRef]

18. Yang, X.; Ren, C.; Xu, H.; Ma, Y.; Shao, F. Transport of ultraintense laser-driven relativistic electrons in dielectric targets. High Power Laser Sci. Eng. 2020, 8, e2. [CrossRef]

19. Guardalben, M.J.; Barczys, M.; Kruschwitz, B.E.; Spilatro, M.; Waxer, L.J.; Hill, E.M. Laser-system model for enhanced operational performance and flexibility on OMEGA EP. High Power Laser Sci. Eng. 2020, 8, e8. [CrossRef]

20. Kim, J.; Bae, J.; Han, Y.; Kim, S.; Jeong, J.; Lee, S. Effectively tun-able dispersion compensation based on chirped fiber Bragg gratings without central wavelength shift. IEEE Photon. Technol. Lett. 2004, 16, 849-851. [CrossRef]

21. Park, H.S.; Hurricane, O.A.; Callahan, D.A.; Casey, D.T.; Dewald, E.L.; Dittrich, T.R.; Döppner, T.; Hinkel, D.E.; Hopkins, L.B.; Pape, S.; et al. High-adiabat high-foot inertial confinement fusion implosion experiments on the National Ignition Facility. Phys. Rev. Lett. 2014, 112, 055001. [CrossRef] [PubMed]

22. Temporal, M.; Canaud, B.; Garbett, W.J.; Ramis, R.; Weber, S. Irradiation uniformity at the Laser MegaJoule facility in the context of the shock ignition scheme. High Power Laser Sci. Eng. 2014, 2, e8. [CrossRef]

23. Huang, C.; Lu, X.; Jiang, Y.; Wang, X.; Qiao, Z.; Fan, W. Real-time characterization of FM-AM modulation in a high-power laser facility using an RF-photonics system and a denoising algorithm. Appl. Opt. 2017, 56, 1610-1615. [CrossRef] [PubMed]

24. Hocquent, S.; Lacroix, G.; Pennincks, D. Compensation of frequency modulation to amplitude modulation conversion in frequency conversion systems. Appl. Opt. 2009, 48, 2515-2521. [CrossRef] [PubMed] 